OPEN ACCESS

Edited by:

Hubing Shi,

Sichuan University, China

Reviewed by:

Lizeng Gao,

Institute of Biophysics (CAS), China

Liu Yang,

China Medical University, China

${ }^{*}$ Correspondence:

Juan Huang

huangjuanxy@med.uestc.edu.cn

${ }^{\dagger}$ These authors share first authorship

Specialty section:

This article was submitted to

Cancer Immunity

and Immunotherapy,

a section of the journal

Frontiers in Immunology

Received: 26 October 2021 Accepted: 22 December 2021

Published: 06 January 2022

Citation:

Tong $\mathrm{H}$, Wei $\mathrm{H}$, Smith $\mathrm{AO}$ and Huang J (2022) The Role of $m 6 A$

Epigenetic Modification in the

Treatment of Colorectal Cancer Immune Checkpoint Inhibitors.

Front. Immunol. 12:802049 doi: 10.3389/fimmu.2021.802049

\section{The Role of m6A Epigenetic Modification in the Treatment of Colorectal Cancer Immune Checkpoint Inhibitors}

\author{
Huan Tong ${ }^{1,2+}$, He Wei ${ }^{3,4 t}$, Alhaji Osman Smith ${ }^{2}$ and Juan Huang ${ }^{1 *}$ \\ 1 Department of Hematology, Sichuan Academy of Medical Sciences \& Sichuan Provincial People's Hospital,University of \\ Electronic Science and Technology of China, Chengdu, China, ${ }^{2}$ Blood Diseases Institute, Xuzhou Medical University, \\ Xuzhou, China \& Department of Hematology, The Affiliated Hospital of Xuzhou Medical University, Xuzhou, China \& Key \\ Laboratory of Bone Marrow Stem Cell, Xuzhou, China, ${ }^{3}$ Department of Gastroenterology, The Second Affiliated Hospital of \\ Chengdu Medical College, Nuclear Industry 416 Hospital, Chengdu, China, 4 School of Bioscience and Technology, \\ Chengdu Medical College, Chengdu, China
}

Tumor immunotherapy, one of the efficient therapies in cancers, has been called to the scientific community's increasing attention lately. Among them, immune checkpoint inhibitors, providing entirely new modalities to treat cancer by leveraging the patient's immune system. They are first-line treatments for varieties of advanced malignancy, such as melanoma, gastrointestinal tumor, esophageal cancer. Although immune checkpoint inhibitors (ICls) treatment has been successful in different cancers, drug resistance and relapses are common, such as in colorectal cancer. Therefore, it is necessary to improve the efficacy of immune checkpoint therapy for cancer patients who do not respond or lowly response to current treatments. N6-methyladenosine (m6A), as a critical regulator of transcript expression, is the most frequently internal modification of mRNA in the human body. Recently, it has been proposed that m6A epigenetic modification is a potential driver of tumor drug resistance. In this report, we will briefly outline the relevant mechanisms, general treatment status of immune checkpoint inhibitors in colorectal cancer, how m6A epigenetic modifications regulate the response of ICls in CRC and provide new strategies for overcoming the resistance of ICls in CRC.

Keywords: colorectal cancer, ICB, resistance, m6A epigenetics, overcome resistance

\section{INTRODUCTION}

The 2018 Global Cancer Statistics estimates the incidence and mortality of 36 cancers in 185 countries around the world. It is reported that the incidence of colorectal cancer is $6.1 \%$, and the mortality rate is $9.2 \%$. The incidence and mortality rate of colorectal cancer is second only to breast cancer in women. In contrast, the incidence and mortality of colorectal cancer in men are second only to lung cancer and prostate cancer (1). In short, the incidence and mortality of colorectal cancer are currently at a high position, which should be paid great attention to and urgently needed effective measures to reduce. In the previous low-incidence areas, the incidence of colorectal cancer showed an upward trend (2). 
Recently, it has been suggested that the patterns and trends of the incidence and mortality of colorectal cancer are connected to human beings' current living standards and lifestyles (3). While China's economic situation is now changing from developing countries to developed countries, Chinese average living standards get remarkably improved. Simultaneously, China is currently also in the stage of cancer transformation, the cancer spectrum is changing toward developed countries, and the cancer burden of colorectal cancer is increasing rapidly (4). Therefore, it is essential to prevent the onset of colorectal cancer and cure colorectal cancer patients, then to improve people's quality of life.

The current management strategies for colorectal cancer mainly include surgery, chemotherapy, radiotherapy, and immunotherapy $(5,6)$. Among them, cancer immunotherapy, containing active immunotherapy, passive immunotherapy, and immune checkpoint inhibitors, is gradually becoming the mainstream of modern cancer treatment (7), which has become a new research direction for cancer treatment and has received extensive attention $(8,9)$.

Recently, tumor immunotherapy, especially in immune checkpoint blockade treatment, has achieved remarkable success in the treatment of colorectal cancer. In particular, immune checkpoint inhibitors have been used in mismatch repair defects and high microsatellite instability (dMMR MSI-H) metastasis, which have been shown to be effective in patients with colorectal cancer and have been approved by the FDA, such as pembrolizumab, nivolumab. Compared with most other treatments for malignant metastatic cancer, immune checkpoint blockade achieved long-term and durable remission in some patients, highlighting the excellent prospects of immune checkpoint blockade in treating colorectal cancer (10-14).

However, with the significant improvement of people's living standards, the quantity of patients with colorectal cancer has continued to hoist, increasing treatment difficulties are found nowadays. It is currently believed that only a tiny proportion of patients with colorectal cancer respond to immune checkpoint inhibitors treatment. While most proficient in mismatch repair (pMMR) and microsatellite stabilization (MSS) or low levels tumors with microsatellite instability (MSI-L) (called pMMR-MSI-L tumors) are ineffective. In these tumors, low tumor mutation burden and lack of immune cell infiltration are thought to be mechanisms of immune resistance $(15,16)$.

Because most pMMR and MSS or MSI-L patients with colorectal cancer is ineffective in ICIs, we wonder that, whether there exist some deeply resistant mechanisms in these patients. It has been proposed that epigenetic modification is a potential driver of tumor drug resistance (17). Since epigenetic inheritance is reversible in nature, the strategy of reversing epigenetic abnormalities is considered to be helpful in treating cancer and reversing drug resistance in cancer therapy (18-20).

In this report, we will briefly outline the relevant mechanisms of tumor immunity, the possible mechanism of tumor ICIs in treating tumors, the current status of colorectal cancer immune checkpoint inhibitors, and a potential method of m6A epigenetic modifications to regulate colorectal cancer ICIs response.

\section{IMMUNOLOGICAL MECHANISMS FOR CANCER}

In human immune system is divided into three categories: the immune defense, immune surveillance, and immune stability, in which tumorigenesis is closely related to the immune surveillance function in the body. Specifically, when the body discovers some cells become cancerous, the immune system will develop an innate immune response and an adaptive immune response targeting to tumor cells or antigens. These immune effector mechanisms influence and regulate each other to achieve the removal of tumor cells within the body (21-25).

In this process, cancer cells secrete some cytokines, which will stimulate the maturation of immature antigen-presenting cells, such as dendritic cells. Mature antigen-presenting cells then present relevant antigens to $\mathrm{CD} 4+$ or $\mathrm{CD} 8+\mathrm{T}$ cells to make them respond, such as secreting some cytokines, which can further act on cytotoxic lymphocytes, Natural killer Cells, macrophages, and enhance the killing of cancer cells, as shown in Figure 1A.

However, even due the body has a series of intelligent immune surveillance, immune clearance of tumor cells, tumor cells escape the body's immune response and weaken the immune system, that result in tumor immune escape mechanism $(21,25)$.

Regarding the tumor immune escape mechanism, the current standard views are as follows: a. The immunogenicity of tumor cells is weakened or missing, b. Tumor antigen modulation, that is, due to the body's immune response to tumor antigens, tumor cell surface antigens are reduced, weakened, or disappear, thus leaving the immune system unrecognizable, allowing the tumor cells to escape the host immune attack, c. tumor cell surface antigens are covered or blocked, d. tumor antigens induce immune tolerance, e. tumor cells would resist apoptosis and induce immune cell apoptosis through Fas/FasL, PD-1/PD-L1 pathways, tumor cells induce immune suppression $(21,25,26)$ as displayed in Figure 1B.

\section{TUMOR IMMUNE CHECKPOINT INHIBITORS}

Given that the body's immune system plays a vital role in the development and progression of tumors, tumor immunotherapy utilizing the body's own immune system to fight against tumor cells is booming. Immunotherapy is expected to become a new development following surgery, chemotherapy, radiotherapy, and targeted tumor therapy as a generation of tumor treatment methods $(5,6)$. The goal of tumor immunotherapy is to release the negative regulatory mechanism in the body and then enhance the immune response of cancer immunity (27). A typical example is to block PD-1/PD-L1 or CTLA-4.

Both PD-1 and CTLA-4 are checkpoints that co-inhibit signaling, which controls $\mathrm{T}$-cell activities, such as activation, proliferation. Immune checkpoint inhibitors, such as PD-1/PDL1 inhibitors and CTLA-4 inhibitors, is a way to remove tumor cells from the inhibitory effect of tumor cells on the immune 

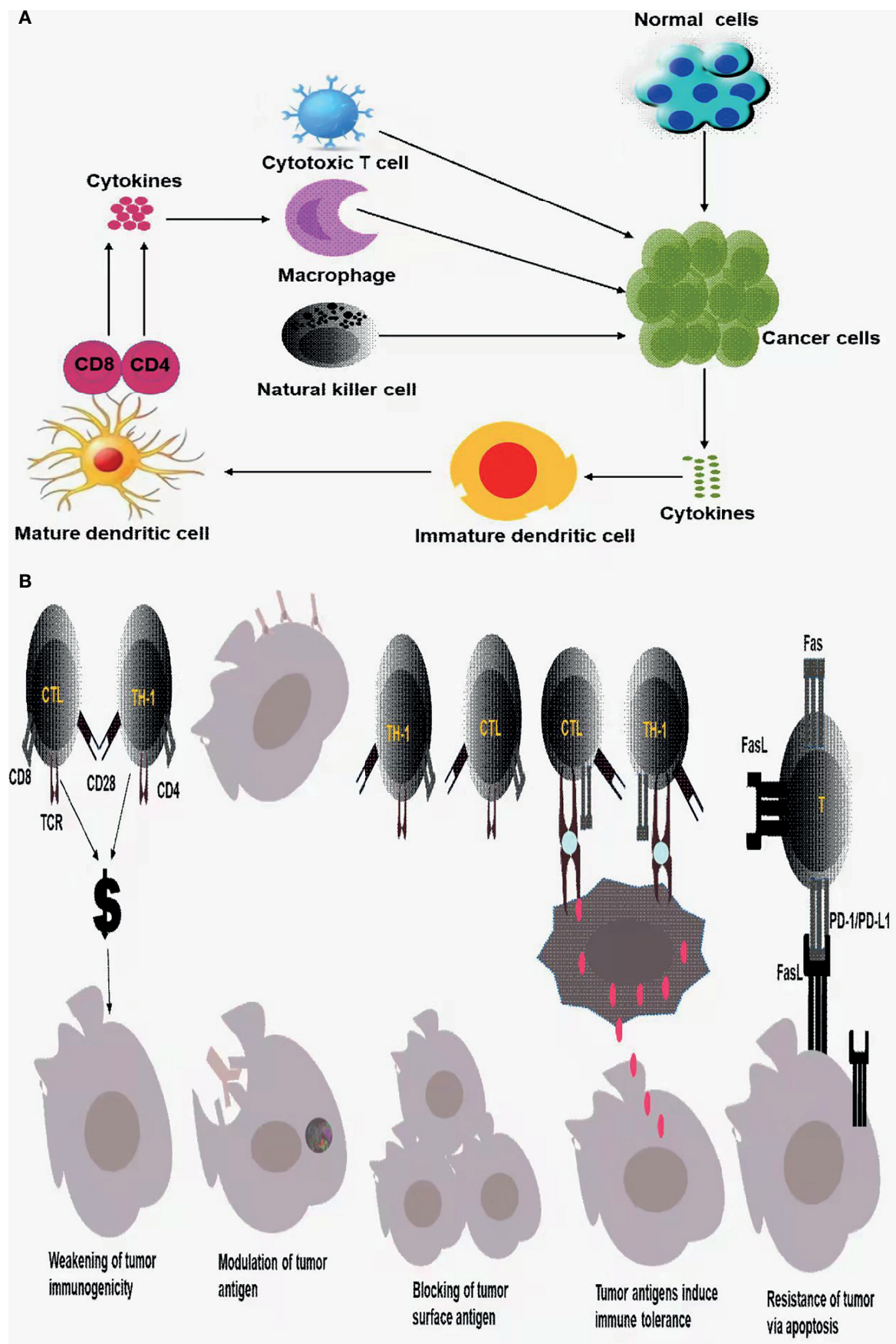

FIGURE 1 | (A) Innate immune response and adaptive immune response to tumor cells; (B) tumor immune escape mechanism.

system, release immune responses, and eliminate tumor cells, which are the excellent content of immunotherapy for colorectal cancer. On the surface of natural killer cells, dendritic cells, B cells, T cells, macrophages, MDSCs, et al. there exists substantial receptors-PD-1(PDCD1/CD279) (28-30). However, PD-1 is usually not expressed in inactivated T cells. PD-1 are not highly expressed until $\mathrm{T}$ cells are activated. The ligand-PD-
L1/PD-L2, is a transmembrane protein that can bind to PD-1 and then negatively regulate $\mathrm{T}$ cell function, specifically, $\mathrm{T}$ cells' activation, proliferation, and survival (31). In tumor microenvironment, tumor cells and tumor-associated APCs highly express PD-L1, while tumor-infiltrating lymphocytes are gradually rich in PD-1 expression under long-term stimulation of tumor antigens. The combination of PD-L1 and PD-1 can 
induce $\mathrm{T}$ cell apoptosis, disability, and depletion (Figure 2), thereby inhibiting the activation, proliferation, and anti-tumor function of tumor antigen-specific CD8+ T cells and achieving tumor immune escape (32-39). Noticeably, the use of PD-1/PDL1 inhibitors would block the PD-1 signaling pathway, restore the body's tumor immunity to normal, and eliminate tumor cells in the body.

CTLA-4(CD152), is a transmembrane protein, whose receptors are $\mathrm{B} 7-1(\mathrm{CD} 80)$ and $\mathrm{B} 7-2(\mathrm{CD} 86)$. CTLA-4 and CD28 share the same receptor, but each of them in the body plays a completely different role. Triggering CD28 will strongly up-regulate $\mathrm{T}$ cells function, enhancing $\mathrm{T}$ cell activation and cytokine production, while triggering CTLA-4 wouldn't not only produce the same effect, but may also down-regulate CD28mediated effects. Generally speaking, CD28 is expressed on resting and activated T cells while CTLA-4 is only expressed on activated T cells. Furthermore, CTLA-4 has a stronger affinity for B7 molecules than CD28 (40). After the TCR-CD3 complex is formed, CTLA-4 will rapidly up-regulate and bind with its high affinity to the co-activated receptors CD80 and CD86 expressed on antigen-presenting cells, thus negatively regulates the activation and function of $\mathrm{T}$ cells (41-47). In the tumor microenvironment, the expression of CTLA-4 in tumor infiltrating regulatory $\mathrm{T}$ cells (Treg) is increased (38, 48-50),

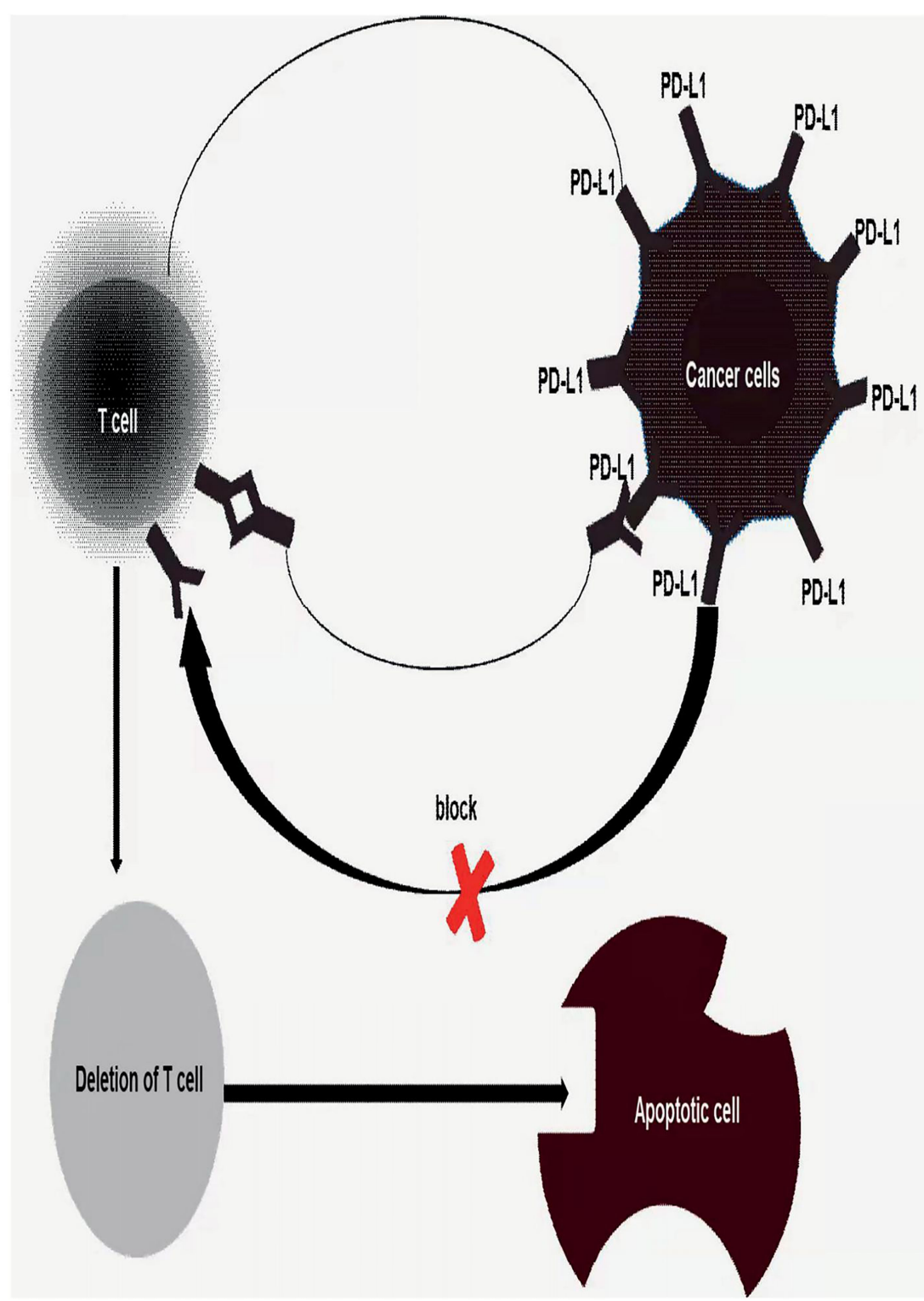

FIGURE 2 | Once tumor's PD-L1 binds to T cells's PD-1 induced depletion, disability and apoptosis of T cells, achieves tumor immune escape. Thus, block the binding of PD1/PD-L1 would be an effective treatment for human cancer or inhibiting tumor proliferation and metastasis. 
thereby inhibiting the activation of tumor antigen-specific $\mathrm{T}$ cells, proliferation and anti-tumor function, realize tumor escape. Unlike PD-1/PD-L1 inhibitors, the using of CTLA-4 inhibitors would like to relieve the inhibitory effect of regulatory $\mathrm{T}$ cells (Treg) in the tumor microenvironment, enhancing the body's tumor immunity to eliminate tumor cells in the body.

Inhibition of PD1/PD-L1 and/or CTLA, beyond all doubt, would become an effective therapy to treat human cancers including colorectal cancer or to inhibit tumor proliferation and metastasis.

\section{ICIS TREATMENT FOR COLORECTAL CANCER}

Starting from this mechanism of tumor immune escape, inhibit the binding of tumor PD-L1 and T cell surface PD-1, block negative regulatory signals, restore the normal immune response of $\mathrm{T}$ cells to tumor cells to realize the role of removing tumor cells. Based on this mechanism, a large number of colorectal cancer patients have now been treated with ICIs. Fortunately, in treating colorectal cancer patients with PD-1, PD-L1, and CTLA4 inhibitors, patients with high microsatellite stability (MSI-H) and mismatch repair defects showed fine efficacy $(10-14,51,52)$. Recent researches demonstrated that Cytotoxic T cells, memory T cells, Th1 cells, N.K. Cells, TFH cells, B cells, activated and mature D.C., M1 macrophages, FOXP $3{ }^{1 \text { ow } C D 45 R A-~ T r e g ~ c e l l s, ~}$ high PD-L1 expression on the exterior of immune cells were confirmed to be strongly associated with a favorable prognosis of CRC (53-59), while CRC patients with massive infiltration of FOXP $3^{\text {hi- }}$ Treg cells, Th17 cells, M2 macrophages, MDSCs, LAMP3 DC and neutrophils have generally a poor prognosis (60-63). Therefore, by raising the level of cells associated with good prognosis of colorectal cancer or lowering the level of cells associated with poor prognosis of colorectal cancer, it may improve the efficacy of colorectal cancer patients. Besides, one of the evaluation indicators over ICIs treatment response is the quantification of circulating tumor DNA (ctDNA). As a practical predictive approach, this indicator can be used in early monitoring to assess the tumor response of patients receiving anti-PD-1 therapy (64). Furthermore, it has shown that MSI, tumor mutation burden(TMB), and MMR played a critical role in the ICB response of colorectal cancer. Generally speaking, CRC patients with MSI-H, high TMB, and dMMR are considered to have a positive reaction to ICIs treatment. Thus CRC patients can get a considerably brilliant prognosis (65-67).

In 2010, Julie R Brahmer, et al. first reported anti-PD-1 in 39 patients with different advanced maglicies, in which one CRC receiving durable complete remission, while other cancers, like melanoma, renal cell cancer did not meet the PR criteria but had significant tumor lesion regression (68). With the in-depth study of anti-immune checkpoint treatment of cancer subsequent studies showed significant activity of PD-1 blockers in multiple cancers, leading to clinical approval for the treatment of multiple tumors, such as gastric cancer (69), colorectal cancer (70), liver cancer (71), and pancreatic cancer (72-74). However, despite of abundant success in treating CRC, there still exists resistance to ICIs in colorectal cancer. The results of current clinical studies are quite explorable, which encourage us to further explore the underlying mechanism of non-response or low-response to ICIs for CRC patients.

Thus, varieties of researches including basic and clinical are done over enhancing CRC efficiency. Researchers made a conclusion that CRC patients with MSI-H, high TMB and dMMR are demonstrated to get a nice response to ICIs treatment and own a considerably brilliant prognosis (65-67, 75-77). Following, FDA approved pebrolizumab used for the treatment of refractory, metastatic solid tumors with mismatch repair defects, high microsatellite instability, and applied nivolumab approval for the treatment of colorectal cancer with a mismatch repair defect and high microsatellite instability.

However, although this colorectal cancer type with very good response to ICIs treatment, there may still exist some resistance and patients still have poor response to ICIs treatment. Romain Cohen, et al. analyzed 38 colorectal cancer patients diagnosed with MSI or dMMR and treated with ICIs and found five had ICB resistance, immediately after they reassessed the status of MSI or dMMR and found that three mCRC patients are characterized with MSS or pMMR (78) showing that MSI-H or dMMR mCRC is misdiagnosed into MSS or pMMR as MSI-H or dMMR status. Therefore, it appears crucial to make a correct diagnosis and to determine MSS and MMR status by immunohistochemistry or PCR before ICIs treatment. Besides, Carino Gurjao, et al. reported that a patient with MSI-H and extremely high neoantigen load found continued disease progression despite with ICIs treatment. Then they tested the intrinsic resistance of $\mathrm{CRC}$ by testing the genomic, transcriptomes, immunohistochemistry of patient tumors and related immune microenvironment. They found that the possible reasons for intrinsic resistance to MSI-CRC were biallelic deletion of B2M (associated with antigen presentation) and increasing infiltration of NK cells and M2 macrophage (79). Therefore, attempting to improve B2M biallelic deletion status and reducing NK cell and M2 macrophage infiltration are possible effective pathways to improve intrinsic resistance to MSI-CRC. Next, CRC patients with MSI-H or dMMR with BRAF mutations also have relatively poor prognosis (80-82), therefore, such patients should be specific analysis, and utilize different therapeutic targets or combination therapy may be needed to improve efficacy.

Unfortunately, CRC patients with MSI-H or dMMR who respond well to ICIs efficacy account for only $10-15 \%$ of the entire CRC patient population, and approximately $85-90 \%$ of CRC patients belongs to the MSS or pMMR type. The current general view is that $\mathrm{mCRC}$ tumor cells accompanied by $\mathrm{pMMR}$ or MSS features have reduced immunogenicity to CD $8+\mathrm{T}$ cells, reduced tumor cell mutation load, reduced HLA class molecule expression, and missing B2M protein are the possible reasons for their poor or even no response to ICIs treatment. There is no doubt that it's urgent to improve the efficacy of CRC patients 
with MSS or pMMR to significantly improve the quality of life of CRC patients.

It is widely accepted that pMMR or MSS mCRC tumor cells have reduced immunogenicity, lower mutational load, low expression of HLA class molecules, and loss of 2heterococytoprotein may be the main reason of (83) for its low or non-response to ICIs treatment. An increasing quantity of researchers have invested basic and clinical research to improve ICIs treatment response in pMMR or MSS mCRC patients, in order to improve progression-free survival and improve their quality of life in CRC patients. They found that ICIs combined with Fruquitinib or Regorafenib (an antiangiogenic drug, the former combination works better than the latter and could significantly improve the progression-free survival of mCRC patients) (84), LDH-A inhibitors (85), MEK inhibitors $(86,87)$, celecoxib (an inhibitor of the cyclooxidase COX2) (88), TGF- $\beta$ inhibitors (89), CXCR4 inhibitors (90) combined treatment of pMMR or MSS mCRC, could significantly improve the treatment response of such patients to ICB in response, which provides a vision of a promising cure for CRC, however, drugs partly produce some toxic side effects such as colitis and duodenal ulcer, one of the pathogenic factors in colorectal cancer. Therefore, there is an urgent need to explore an effective treatment of CRC patients, including MSI-H or dMMR characteristics and PMMR or MSS characteristics, to improve progression-free survival and improve their quality of life in CRC patients.

\section{M6A EPIGENETIC MODIFICATION}

Current studies have shown that epigenetic defects played a crucial role in all malignant tumors with genetic defects $(18,19$, 91, 92). Cancer is a disease caused by the continuous accumulation of genetic and epigenetic alteration. Generally, epigenetic alteration often precedes cancer and then induce gene mutations which lead to cancer (93). Almost all human cancer types contain epigenetic alterations. Because epigenetic modifications are reversible in nature (18-20), epigenetics has become an attractive target for cancer therapy. Recent studies have found that, in addition to being related to cancer progression, epigenetic modification is also a potential driver of drug resistance (17).

N6-methyladenosine (m6A) modification is the most common epigenetic methylation modification in RNA, such as mRNAs, ncRNAs, approximately $80 \%$ of all RNA methylation modification in vivo (94), which influences RNA function, for example, it has an great effect on RNA splicing, export, stability and translation. m6A modification is a reversible and dynamic in RNA epigenetic process. It is adjusted by m6A regulators, the main regulators containing "writer" (methyltransferase), "reader" (signal converter), combined with "eraser" (demethylation basease) (95).

Specifically, the writers, whose function is adding methylation into RNA to make the RNA methylated, up to date, are composed of METTL3, METTL14, WTAP, RBM15, RBM15B, ZC3H13 and KIAA1429 (96, 97). The readers, identifying methylation sites, are contained with YTHDC1, YTHDC2, YTHDF1, YTHDF2, YTHDF3, HNRNPC,IMP2 and HNRNPA2B1 (98-101). The erasers, whose role is deleting the methylation of RNA, mainly consist of FTO and ALKBH5 (102, 103) as revealed in Figure 3.

\section{m6A MODIFICATION, ICIs IN CRC}

At present, anti-immune checkpoint treatment of CRC is the thoroughly studied type of immunotherapy and has left some treating troubles so far (17). Researches lately have displayed that, in addition to being related to cancer progression, m6 A epigenetic modification is also a potential driver of drug resistance (104). There are several main mechanisms that lead to anti-cancer drug resistance: changes in drug metabolism, deregulation of drug transport, mutations or changes in target protein expression, the above-mentioned mechanisms are the main reasons for the decrease in the efficacy of anti-cancer drugs (105-109).

Among several new methods to achieve the goal of drug sensitive, the regulation of m6A RNA modification has been demonstrated to be an overwhelming strategy for the drug resistance of various types of cancer cells. M6A regulates RNA's different-stage function, such as RNA splicing, degradation and translation to take part in a variety of biological processes including cell proliferation, metabolism and metastasis, and the caused anti-tumor resistance (109).

Immune checkpoint inhibitors have been utilized to colorectal cancer therapy and provides a promising clinical advantage; however, it usually shows that CRC patients make a low response or resistance (110). To improve the efficacy of ICB, it is urgently needed to identify the underlying mechanism for the low-response or resistance of ICIs in CRC. Recent studies have shown that m6A modifications are highly related with the low-response or resistance of ICIs in CRC, one possible reason that m6A tightly connected with microenvironment of tumor and immune cells in CRC.

The m6A modification pathway is often misregulated in cancer (111). Bo Zhang, et al. revealed that m6A modification is necessary in TME (112-115). The high m6A score, characterized by decreased mutation burden and immune activation, is related to reduced neoantigen load and poor response to ICIs (115). Patients with higher m6A-related risks have lower expression of immune checkpoint molecules, indicating that ICIs treatment may be less efficient for this subgroup. Therefore, m6A risk characteristics could be used as immunotherapy predictors for STAD prognosis in a clinical setting, and the underlying mechanism of the relationship between immune checkpoint molecules and m6A methylation needs to be further studied (115). In the same way as gastric cancer, does this apply to the colorectal tumors belonging to the digestive tract tumor? This will provide new ideas for clinically predicting the ICIs response of patients with colorectal cancer and further strengthening ICIs therapeutic response in colorectal cancer. 


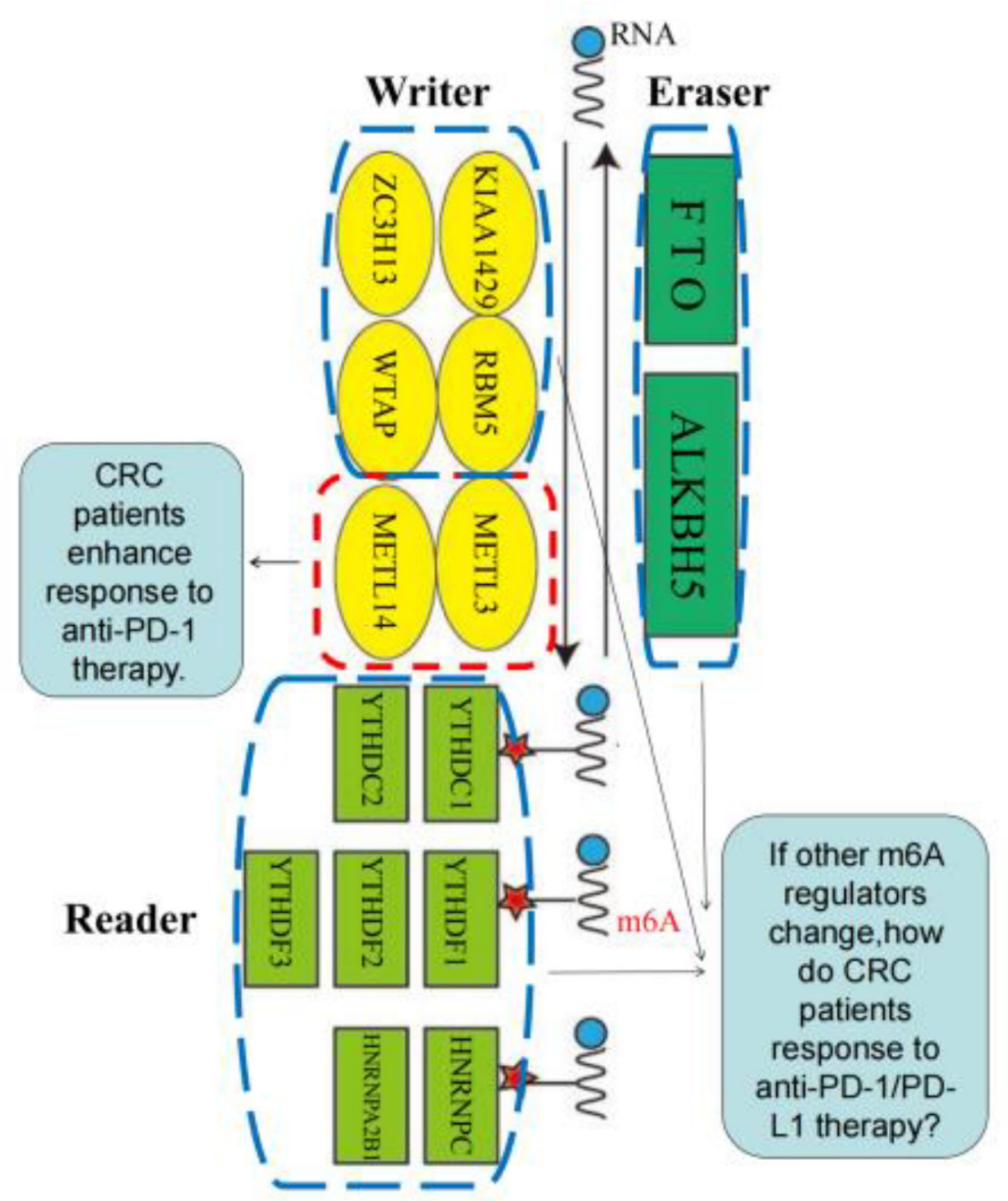

FIGURE 3 | m6A regulators, including "writer" (methyltransferase), "reader" (signal converter) and "eraser" (demethylation basease).

\section{m6A Writers, ICls in CRC}

M6A modification regulated by METTL3 may promotes D.C. activation and maturation, Specific knockout Mettl3 in D.C. causes impaired phenotypic and functional maturation of D.C. and decreases their capacities of stimulating $\mathrm{T}$ cell responses (112). Specific depletion of Mettl3 in tumorassociated macrophages(TAM) resulted in CD8+ T cells dysfunction and tumor growth (113). With the reducing of m6A regulated by Mettl14, expression of the tumor suppressor KLF4 will be substantially increased and will further promote the migration of CRC cells and invasion (114). Interestingly some reseachers believed that m6A writers, such as Mettl3, WTAP, is positively associated with CRC cells invasion, migration, progression, and tumor stem cells with stemness and drug resistance (116-119).

Chen Xiaoxiang, et al. explored the function of m6A modification in CRC, illustrated the mechanism of m6A modification involved in the biological process of CRC, and confirmed that METTL14 is related to the progression of CRC in vivo and in vitro (112-114, 116-120). Lingling Wang et al. found that the deletion of methyltransferases Mettl3 and Mettl14 inhibited N6 methyladenosine (m6A) mRNA modification, enhanced pMMR-MSI-L colorectal cancer and melanoma patients' response to anti-PD-1 therapy, and significantly slowed down tumor grows and prolongs the patient's survival. In addition, the deficiency of MettL3 or Mettl14 in tumors results in enhancing cytotoxic tumor-infiltrating CD8+ T cells, and increasing the secretion of IFN-c, Cxcl9 and Cxcl10 in TME in vivo, which proves that the immune system and tumor microenvironment have metastasized in tumor m6A mRNA. Changes will occur after the enzyme is removed. In patients with colorectal cancer and melanoma undergoing immunotherapy, changes in the transcriptome profile of methyltransferasedeficient tumors in tumor cells indicate that the activation of 
IFN-c signaling is the key to resensitization, while external transcriptome analysis shows that IFN -c-Stat1-Irf1 axis transcript lacking m6A modification contributes to the stabilization of $\mathrm{m} 6 \mathrm{~A}$ reader Ythdf2-mediated transcripts, thereby explaining the up-regulation of IFN-c signaling and changes in the tumor microenvironment (121), this discovery will further promote the understanding and in-depth study of m6A methyltransferase in the anti-PD-1/PD-L1 treatment of colorectal cancer.

However, whether and how m6A modifications writers alteration influence the efficiency of ICIs in colorectal cancer, still remains further research.

\section{m6A Erasers, ICls in CRC}

Zeyan Zhang, et al. studied m6A erasers and found that reducing FTO expression, inhibiting MZF1 expression, and thus c-Myc expression and hindered CRC cell proliferation and progression (122). Contrary to the conclusion, Danyun Ruan, et al. and Sebastien Relier, et al. suggested that inhibiting FTO expression would promote CRC metastasis, poor prognosis and high recurrence in CRC patients $(101,123)$.

The study by Seungwon Yang, et al. found that under the action of the demethylase FTO, m6A will promote the growth of melanoma and reduce its response to anti-PD-1 blocking immunotherapy. When FTO is knocked out, it can increase m6A methylation in key tumorigenic melanoma cells (including PD-1, CXCR4 and SOX10), resulting in increased RNA attenuation through $\mathrm{m} 6 \mathrm{~A}$ reader YTHDF2, and then cause that melanoma cells are sensitive to interferon- $\gamma($ IFN- $\gamma$ ) and make melanoma sensitive to anti-PD-1 treatment. It is generally believed that inhibiting the combination of FTO and anti-PD-1 blockade may attenuate the resistance of melanoma to immunotherapy and improve the treatment response (124). However, they did not conduct research on colorectal cancer like Lingling Wang et al., but this will open a new way of thinking about ICIs resistance in colorectal cancer patients.

Then the study by Na Lia, et al. found that the knockout of the m6A demethylase ALKBH5 would make tumors susceptible to cancer immunotherapy. Specifically, ALKBH5 regulates the expression level and lactic acid content of Mct4/Slc16a3 in the TME and the constitution of tumor infiltrating Tregs and myeloid-derived suppressor cells. The research results show that $\mathrm{m} 6 \mathrm{~A}$ demethylase in tumor cells contributes to the effect of immunotherapy, and ALKBH5 is identified as a potential therapeutic target to improve the efficacy of immunotherapy for melanoma, colorectal cancer and other potential cancers (125).

However, whether and how m6A modifications erasers variations influence the efficiency of ICIs in colorectal cancer, still remains further research.

\section{m6A Readers, ICls in CRC}

Numerous studies on m6A Reader have confirmed four genes (YTHDF1, IGF2BP1, IGF2BP1, IGFBP3, EIF3B) is a potential biomarker of CRC. It was found to downregulate YTHDF1 or IMP2, can further regulate the GLS1-glutamine metabolic axis, improve the stability of the m6A-modified GSK3 $\beta$ mRNA, inhibition of Wnt/-catenin/cyclin D1 expression, inhibition of
CRC cell proliferation, colony formation, and increase the apoptosis levels in CRC cells, To sensitized cisplatin-resistant CRC cells (126-128).

Kazuo Tsuchiya, et al. used immunohistochemistry to detect the protein expression levels of $\mathrm{m} 6 \mathrm{~A}$ readers, such as YTHDF1 and YTHDF2 in 603 cases of non-small cell lung cancer tissues and evaluated four subgroups as TILs in tumor nests and surrounding stroma (PD-1, CD8, Foxp3 and CD45RO), and to study the differential expression of PD-L1 in lung cancer cells lacking YTHDF1 and YTHDF2. They found that YTHDF1 and YTHDF2 lowerly expressed in advanced tumors than that of early tumors, and the expression of YTHDF1 and YTHDF2 were also advised to be independent favorable prognostic factors for recurrence-free survival. In tumors with high expression of YTHDF1 and YTHDF2, the TIL density of almost all four lymphocyte subgroups in the stroma was significantly increased. In vitro, YTHDF1 and YTHDF2 knockout in cells up-regulated the expression of tumor PD-L1 and changed a variety of immune-related genes. It shows that the high expression of YTHDF1 and YTHDF2 is related to the good prognosis of NSCLC patients, the increase in the number of TIL and the down-regulation of PD-L1. YTHDF1 and YTHDF2 may be new prognostic and drug targets related to lung cancer tumor immune microenvironment (129). However, whether the upregulation of YTHDF1 and YTHDF2 is effective for patients with colorectal cancer needs further research.

However, whether and how m6A modifications readers changes influence the efficiency of ICIs in colorectal cancer, still remains further research.

The possible reasons for such interestingly different and even opposite conclusions for different researchers in the same study subject maybe depended on m6A modified different RNA sites, different patterns in different immune cells, or different types of CRC, for example, MSS/pMMR or MSI-H/dMMR and whether accompanied by BRAF mutations.

However, due to methodological limitations, these studies are limited to one or two RNA modification "writers", "erasers" or "readers", but the antitumor effects of RNA modifications are characterized by highly complicated interactions of many regulators. Thus, a comprehensive and full understanding of how the regulatory networks of multiple RNA modification, there is no doubt that, will absolutely help us to have a better understanding of immunomodulatory and development of immunotherapy strategies in low-response or resistance of ICIs in CRC patients.

\section{CONCLUSIONS AND PROSPECTS}

The success of tumor immunotherapy, especially the success of ICIs treatment, has brought encouraging hope and confidence for better treatment and possible cure of colorectal cancer patients. However, because about $85-90 \%$ of colorectal cancer patients have the characteristics of pMMR or MSI-L in clinical practice, whose typical feature is that the tumor with a lower mutation burden has a poor curative effect, usually showing resistance to ICIs treatment. So this will force us to further 
explore the cure of colorectal cancer and the mechanism of PD1/PD-L1 resistance.

In this review, we summarized the mechanism of immune escape in the process of general cancer and the current status of immune checkpoint blockade treatment of colorectal cancer. At the same time, we also deeply explored and guess the role of m6A in mechanism of resistance in colorectal cancer anti-PD-1/PDL1 therapy.

For the brief mechanism of $\mathrm{m} 6 \mathrm{~A}$ to relieve the resistance of colorectal cancer patients to anti-PD-1/PD-L1 therapy, we summarize the existing research and speculate the following findings. The m6A modification regulated by the $\mathrm{m} 6 \mathrm{~A}$ regulator includes the "writer" (methyltransferase_METTL3, METL14), "reader" (signal converter_YTHDC1, YTHDC2) and "eraser" (demethylase_FTO, ALKBH5) (67). Inhibition of "writers" (methyltransferase_Mettl3, Mettl14) can inhibit m6A modification, enhance the response of pMMR-MSI-L colorectal cancer patients to anti-PD-1 treatment, and significantly slow down tumor growth and prolong patient survival; activation the high expression of "readers" (signal converters_YTHDF1 and YTHDF2) may be related to the good prognosis of patients with colorectal cancer, the increase in the number of TIL, and the down-regulation of $\mathrm{PD}-\mathrm{L} 1$, and may become a novel related to the immune microenvironment of colorectal cancer. The prognosis and drug targets of the drug; inhibiting the "eraser"

\section{REFERENCES}

1. Bray F, Ferlay J, Soerjomataram I, Siegel RL, Torre LA, Jemal A. Global Cancer Statistics 2018: GLOBOCAN Estimates of Incidence and Mortality Worldwide for 36 Cancers in 185 Countries. CA Cancer J Clin (2018) 68 (6):394-424. doi: 10.3322/caac. 21492

2. Arnold M, Abnet CC, Neale RE, Vignat J, Giovannucci EL, McGlynn KA, et al. Global Burden of 5 Major Types of Gastrointestinal Cancer. Gastroenterology (2020) 159(1):335-349.e15. doi: 10.1053/j.gastro.2020.02.068

3. Arnold M, Sierra MS, Laversanne M, Soerjomataram I, Jemal A, Bray F. Global Patterns and Trends in Colorectal Cancer Incidence and Mortality. Gut (2017) 66(4):683-91. doi: 10.1136/gutjnl-2015-310912

4. Feng RM, Zong YN, Cao SM, Xu RH. Current Cancer Situation in China: Good or Bad News From the 2018 Global Cancer Statistics? Cancer Commun (Lond) (2019) 39(1):22. doi: 10.1186/s40880-019-0368-6

5. Mármol I, Sánchez-de-Diego C, Pradilla Dieste A, Cerrada E, Rodriguez Yoldi MJ. Colorectal Carcinoma: A General Overview and Future Perspectives in Colorectal Cancer. Int J Mol Sci (2017) 18(1):197. doi: 10.3390/ijms 18010197

6. Tang Y, Zhang T, Zhou X, Zhao Y, Xu H, Liu Y, et al. The Preoperative Prognostic Value of the Radiomics Nomogram Based on CT Combined With Machine Learning in Patients With Intrahepatic Cholangiocarcinoma. World J Surg Oncol (2021) 19(1):45. doi: 10.1186/s12957-021-02162-0

7. Li B, Cui Y, Nambiar DK, Sunwoo JB, Li R. The Immune Subtypes and Landscape of Squamous Cell Carcinoma. Clin Cancer Res (2019) 25 (12):3528-37. doi: 10.1158/1078-0432.CCR-18-4085

8. Finck A, Gill SI, June CH. Cancer Immunotherapy Comes of Age and Looks for Maturity. Nat Commun (2020) 11(1):3325. doi: 10.1038/s41467-020-17140-5

9. Liu Z, Ravindranathan R, Kalinski P, Guo ZS, Bartlett DL. Rational Combination of Oncolytic Vaccinia Virus and PD-L1 Blockade Works Synergistically to Enhance Therapeutic Efficacy. Nat Commun (2017) 8:14754. doi: 10.1038/ncomms14754

10. Schreiber RD, Old LJ, Smyth MJ. Cancer Immunoediting: Integrating Immunity's Roles in Cancer Suppression and Promotion. Science (2011) 331(6024):1565-70. doi: 10.1126/science.1203486
(demethylase_FTO, ALKBH5) may increase the sensitivity of colorectal cancer patients to immune checkpoint therapy and reduce resistance after the anti-PD-1/PD-L1 therapy.

At present, in colorectal cancer ICIs treatment resistance research, only Lingling Wang et al. from the $\mathrm{m} 6 \mathrm{~A}$ "author" (methyltransferase_Mettl3, Mettl14) point of view, found that the deletion of methyltransferase Mettl3 and Mettl14 inhibits N6 methyladenosine (m6A) mRNA modification, enhances the response of pMMR-MSI-L colorectal cancer and melanoma patients to anti-PD-1 treatment, and significantly slows tumor growth and prolongs patient survival. And whether m6A "reader" (signal converter_YTHDF1, YTHDF2), "eraser" (demethylase_FTO, ALKBH5), etc. or how to convert can increase the sensitivity of colorectal cancer patients to immune checkpoint therapy and reduce the resistance of colorectal cancer patients to anti-PD-1/PD-L1 therapy still needs further research.

\section{AUTHOR CONTRIBUTIONS}

HT, HW, and AS made significant contribution on writing this review. JH designed the topic and checked the manuscript. All authors contributed to the article and approved the submitted version.

11. Wang S, He Z, Wang X, Li H, Liu XS. Antigen Presentation and Tumor Immunogenicity in Cancer Immunotherapy Response Prediction. Elife (2019) 8:e49020. doi: 10.7554/eLife.49020

12. Schmid D, Park CG, Hartl CA, Subedi N, Cartwright AN, Puerto RB, et al. T Cell-Targeting Nanoparticles Focus Delivery of Immunotherapy to Improve Antitumor Immunity. Nat Commun (2017) 8(1):1747. doi: 10.1038/s41467017-01830-8

13. Ciardiello D, Vitiello PP, Cardone C, Martini G, Troiani T, Martinelli E, et al Immunotherapy of Colorectal Cancer: Challenges for Therapeutic Efficacy. Cancer Treat Rev (2019) 76:22-32. doi: 10.1016/j.ctrv.2019.04.003

14. Ganesh K, Stadler ZK, Cercek A, Mendelsohn RB, Shia J, Segal NH, et al. Immunotherapy in Colorectal Cancer: Rationale, Challenges and Potential. Nat Rev Gastroenterol Hepatol (2019) 16(6):361-75. doi: 10.1038/s41575019-0126-x

15. Galon J, Costes A, Sanchez-Cabo F, Kirilovsky A, Mlecnik B, Lagorce- Pagès C, et al. Type, Density, and Location of Immune Cells Within Human Colorectal Tumors Predict Clinical Outcome. Science (2006) 313 (5795):1960-4. doi: 10.1126/science.1129139

16. Le DT, Durham JN, Smith KN, Wang H, Bartlett BR, Aulakh LK, et al. Mismatch Repair Deficiency Predicts Response of Solid Tumors to PD-1 Blockade. Science (2017) 357(6349):409-13. doi: 10.1126/science.aan6733

17. Adelaiye-Ogala R, Budka J, Damayanti NP, Arrington J, Ferris M, Hsu CC, et al. EZH2 Modifies Sunitinib Resistance in Renal Cell Carcinoma by Kinome Reprogramming. Cancer Res (2017) 77(23):6651-66. doi: 10.1158/ 0008-5472.CAN-17-0899

18. Tomar T, de Jong S, Alkema NG, Hoekman RL, Meersma GJ, Klip HG, et al. Genome-Wide Methylation Profiling of Ovarian Cancer Patient-Derived Xenografts Treated With the Demethylating Agent Decitabine Identifies Novel Epigenetically Regulated Genes and Pathways. Genome Med (2016) 8 (1):107. doi: 10.1186/s13073-016-0361-5

19. Vijayaraghavalu S, Dermawan JK, Cheriyath V, Labhasetwar V. Highly Synergistic Effect of Sequential Treatment With Epigenetic and Anticancer Drugs to Overcome Drug Resistance in Breast Cancer Cells Is Mediated via Activation of P21 Gene Expression Leading to G2/M Cycle Arrest. Mol Pharm (2013) 10(1):337-52. doi: 10.1021/mp3004622 
20. Ahn DH, Javle M, Ahn CW, Jain A, Mikhail S, Noonan AM, et al. NextGeneration Sequencing Survey of Biliary Tract Cancer Reveals the Association Between Tumor Somatic Variants and Chemotherapy Resistance. Cancer (2016) 122(23):3657-66. doi: 10.1002/cncr.30247

21. Mohme M, Riethdorf S, Pantel K. Circulating and Disseminated Tumour Cells - Mechanisms of Immune Surveillance and Escape. Nat Rev Clin Oncol (2017) 14(3):155-67. doi: 10.1038/nrclinonc.2016.144

22. Ricklin D, Hajishengallis G, Yang K, Lambris JD. Complement: A Key System for Immune Surveillance and Homeostasis. Nat Immunol (2010) 11 (9):785-97. doi: 10.1038/ni.1923

23. Swann JB, Smyth MJ. Immune Surveillance of Tumors. J Clin Invest (2007) 117(5):1137-46. doi: 10.1172/JCI31405

24. Gajewski TF, Schreiber H, Fu YX. Innate and Adaptive Immune Cells in the Tumor Microenvironment. Nat Immunol (2013) 14(10):1014-22. doi: $10.1038 /$ ni.2703

25. Kim R, Emi M, Tanabe K. Cancer Immunoediting From Immune Surveillance to Immune Escape. Immunology (2007) 121(1):1-14. doi: 10.1111/j.1365-2567.2007.02587.x

26. Jiang X, Wang J, Deng X, Xiong F, Ge J, Xiang B, et al. Role of the Tumor Microenvironment in PD-L1/PD-1-Mediated Tumor Immune Escape. $\mathrm{Mol}$ Cancer (2019) 18(1):10. doi: 10.1186/s12943-018-0928-4

27. Liu Y, Zheng P. Preserving the CTLA-4 Checkpoint for Safer and More Effective Cancer Immunotherapy. Trends Pharmacol Sci (2020) 41(1):4-12. doi: 10.1016/j.tips.2019.11.003

28. Schwartz RH. T Cell Anergy. Annu Rev Immunol (2003) 21:305-34. doi: 10.1146/annurev.immunol.21.120601.141110

29. Pardoll DM. The Blockade of Immune Checkpoints in Cancer Immunotherapy. Nat Rev Cancer (2012) 12(4):252-64. doi: 10.1038/ nrc3239

30. Finger LR, Pu J, Wasserman R, Vibhakar R, Louie E, Hardy RR, et al. The Human PD-1 Gene: Complete cDNA, Genomic Organization, and Developmentally Regulated Expression in B Cell Progenitors. Gene (1997) 197(1-2):177-87. doi: 10.1016/s0378-1119(97)00260-6

31. Shi L, Chen S, Yang L, Li Y. The Role of PD-1 and PD-L1 in T-Cell Immune Suppression in Patients With Hematological Malignancies. J Hematol Oncol (2013) 6(1):74. doi: 10.1186/1756-8722-6-74

32. Thibult ML, Mamessier E, Gertner-Dardenne J, Pastor S, Just-Landi S, Xerri L, et al. PD-1 is a Novel Regulator of Human B-Cell Activation. Int Immunol (2013) 25(2):129-37. doi: 10.1093/intimm/dxs098

33. Topalian SL, Drake CG, Pardoll DM. Targeting the PD-1/B7-H1(PD-L1) Pathway to Activate Anti-Tumor Immunity. Curr Opin Immunol (2012) 24 (2):207-12. doi: 10.1016/j.coi.2011.12.009

34. Luo Y, Yang J, Yu J, Liu X, Yu C, Hu J, et al. Long Non-Coding RNAs: Emerging Roles in the Immunosuppressive Tumor Microenvironment. Front Oncol (2020) 10:48. doi: 10.3389/fonc.2020.00048

35. Ghebeh H, Mohammed S, Al-Omair A, Qattan A, Lehe C, Al-Qudaihi G, et al. The B7-H1 (PD-L1) T Lymphocyte-Inhibitory Molecule is Expressed in Breast Cancer Patients With Infiltrating Ductal Carcinoma: Correlation With Important High-Risk Prognostic Factors. Neoplasia (2006) 8(3):190-8. doi: $10.1593 /$ neo.05733

36. Wong RM, Scotland RR, Lau RL, Wang C, Korman AJ, Kast WM, et al. Programmed Death-1 Blockade Enhances Expansion and Functional Capacity of Human Melanoma Antigen-Specific CTLs. Int Immunol (2007) 19(10):1223-34. doi: 10.1093/intimm/dxm091

37. Ni Y, Zhou X, Yang J, Shi H, Li H, Zhao X, et al. The Role of Tumor-Stroma Interactions in Drug Resistance Within Tumor Microenvironment. Front Cell Dev Biol (2021) 9:637675. doi: 10.3389/fcell.2021.637675

38. Ahmadzadeh M, Johnson LA, Heemskerk B, Wunderlich JR, Dudley ME, White DE, et al. Tumor Antigen-Specific CD8 T Cells Infiltrating the Tumor Express High Levels of PD-1 and are Functionally Impaired. Blood (2009) 114(8):1537-44. doi: 10.1182/blood-2008-12-195792

39. Fourcade J, Sun Z, Benallaoua M, Guillaume P, Luescher IF, Sander C, et al. Upregulation of Tim-3 and PD-1 Expression is Associated With Tumor Antigen-Specific CD8+ T Cell Dysfunction in Melanoma Patients. J Exp Med (2010) 207(10):2175-86. doi: 10.1084/jem.20100637

40. Boussiotis VA. Molecular and Biochemical Aspects of the PD-1 Checkpoint Pathway. N Engl J Med (2016) 375(18):1767-78. doi: 10.1056/ NEJMra1514296
41. Gordon SR, Maute RL, Dulken BW, Hutter G, George BM, McCracken MN, et al. PD-1 Expression by Tumour-Associated Macrophages Inhibits Phagocytosis and Tumour Immunity. Nature (2017) 545(7655):495-9. doi: 10.1038 /nature22396

42. Linsley PS, Bradshaw J, Greene J, Peach R, Bennett KL, Mittler RS. Intracellular Trafficking of CTLA-4 and Focal Localization Towards Sites of TCR Engagement. Immunity (1996) 4(6):535-43. doi: 10.1016/s10747613(00)80480-x

43. Walunas TL, Lenschow DJ, Bakker CY, Linsley PS, Freeman GJ, Green JM, et al. CTLA-4 can Function as a Negative Regulator of T Cell Activation. Immunity (1994) 1(5):405-13. doi: 10.1016/1074-7613(94)90071-X

44. Fallarino F, Fields PE, Gajewski TF. B7-1 Engagement of Cytotoxic T Lymphocyte Antigen 4 Inhibits T Cell Activation in the Absence of CD28. J Exp Med (1998) 188(1):205-10. doi: 10.1084/jem.188.1.205

45. Krummel MF, Allison JP. CD28 and CTLA-4 Have Opposing Effects on the Response of T Cells to Stimulation. J Exp Med (1995) 182(2):459-65. doi: 10.1084/jem.182.2.459

46. Masteller EL, Chuang E, Mullen AC, Reiner SL, Thompson CB. Structural Analysis of CTLA-4 Function In Vivo. J Immunol (Baltimore Md 1950) (2000) 164,10:5319-27. doi: 10.4049/jimmunol.164.10.5319

47. Peggs KS, Quezada SA, Chambers CA, Korman AJ, Allison JP. Blockade of CTLA-4 on Both Effector and Regulatory T Cell Compartments Contributes to the Antitumor Activity of Anti-CTLA-4 Antibodies. J Exp Med (2009) 206 (8):1717-25. doi: 10.1084/jem.20082492

48. van der Merwe PA, Bodian DL, Daenke S, Linsley P, Davis SJ. CD80 (B7-1) Binds Both CD28 and CTLA-4 With a Low Affinity and Very Fast Kinetics. J Exp Med (1997) 185(3):393-403. doi: 10.1084/jem.185.3.393

49. Qureshi OS, Kaur S, Hou TZ, Jeffery LE, Poulter NS, Briggs Z, et al. Constitutive Clathrin-Mediated Endocytosis of CTLA-4 Persists During T Cell Activation. J Biol Chem (2012) 287(12):9429-40. doi: 10.1074/ jbc.M111.304329

50. Walker LS, Sansom DM. The Emerging Role of CTLA4 as a Cell-Extrinsic Regulator of T Cell Responses. Nat Rev Immunol (2011) 11(12):852-63. doi: $10.1038 /$ nri3108

51. André T, Shiu KK, Kim TW, Jensen BV, Jensen LH, Punt C, et al. KEYNOTE-177 Investigators. Pembrolizumab in Microsatellite-InstabilityHigh Advanced Colorectal Cancer. N Engl J Med (2020) 383(23):2207-18. doi: 10.1056/NEJMoa2017699

52. Le DT, Uram JN, Wang H, Bartlett BR, Kemberling H, Eyring AD, et al. PD1 Blockade in Tumors With Mismatch-Repair Deficiency. $N$ Engl J Med (2015) 372(26):2509-20. doi: 10.1056/NEJMoa1500596

53. Kim HR, Ha SJ, Hong MH, Heo SJ, Koh YW, Choi EC, et al. PD-L1 Expression on Immune Cells, But Not on Tumor Cells, is a Favorable Prognostic Factor for Head and Neck Cancer Patients. Sci Rep (2016) 6:36956. doi: 10.1038/srep36956

54. Bruni D, Angell HK, Galon J. The Immune Contexture and Immunoscore in Cancer Prognosis and Therapeutic Efficacy. Nat Rev Cancer (2020) 20 (11):662-80. doi: 10.1038/s41568-020-0285-7

55. Tosolini M, Kirilovsky A, Mlecnik B, Fredriksen T, Mauger S, Bindea G, et al. Clinical Impact of Different Classes of Infiltrating T Cytotoxic and Helper Cells (Th1, Th2, Treg, Th17) in Patients With Colorectal Cancer. Cancer Res (2011) 71(4):1263-71. doi: 10.1158/0008-5472.CAN-10-2907

56. Coca S, Perez-Piqueras J, Martinez D, Colmenarejo A, Saez MA, Vallejo C, et al. The Prognostic Significance of Intratumoral Natural Killer Cells in Patients With Colorectal Carcinoma. Cancer (1997) 79(12):2320-8. doi: 10.1002/(sici)1097-0142(19970615)79:12<2320::aid-cncr5>3.0.co;2-p

57. Melero I, Rouzaut A, Motz GT, Coukos G. T-Cell and NK-Cell Infiltration Into Solid Tumors: A Key Limiting Factor for Efficacious Cancer Immunotherapy. Cancer Discov (2014) 4(5):522-6. doi: 10.1158/21598290.CD-13-0985

58. Kinouchi M, Miura K, Mizoi T, Ishida K, Fujibuchi W, Sasaki H, et al Infiltration of CD40-Positive Tumor-Associated Macrophages Indicates a Favorable Prognosis in Colorectal Cancer Patients. Hepatogastroenterology (2013) 60(121):83-8. doi: 10.5754/hge12372

59. Malietzis G, Lee GH, Jenkins JT, Bernardo D, Moorghen M, Knight SC, et al Prognostic Value of the Tumour-Infiltrating Dendritic Cells in Colorectal Cancer: A Systematic Review. Cell Commun Adhes (2015) 22(1):9-14 doi: 10.3109/15419061.2015.1036859 
60. Saito T, Nishikawa H, Wada H, Nagano Y, Sugiyama D, Atarashi K, et al. Two FOXP3(+)CD4(+) T Cell Subpopulations Distinctly Control the Prognosis of Colorectal Cancers. Nat Med (2016) 22(6):679-84. doi: $10.1038 / \mathrm{nm} .4086$

61. Aras S, Zaidi MR. TAMeless Traitors: Macrophages in Cancer Progression and Metastasis. Br J Cancer (2017) 117(11):1583-91. doi: 10.1038/bjc.2017.356

62. Mosser DM, Edwards JP. Exploring the Full Spectrum of Macrophage Activation. Nat Rev Immunol (2008) 8(12):958-69. doi: 10.1038/nri2448

63. Sandel MH, Dadabayev AR, Menon AG, Morreau H, Melief CJ, Offringa R, et al. Prognostic Value of Tumor-Infiltrating Dendritic Cells in Colorectal Cancer: Role of Maturation Status and Intratumoral Localization. Clin Cancer Res (2005) 11(7):2576-82. doi: 10.1158/1078-0432.CCR-04-1448

64. Cabel L, Riva F, Servois V, Livartowski A, Daniel C, Rampanou A, et al. Circulating Tumor DNA Changes for Early Monitoring of Anti-PD1 Immunotherapy: A Proof-of-Concept Study. Ann Oncol (2017) 28 (8):1996-2001. doi: 10.1093/annonc/mdx212

65. Eso Y, Shimizu T, Takeda H, Takai A, Marusawa H. Microsatellite Instability and Immune Checkpoint Inhibitors: Toward Precision Medicine Against Gastrointestinal and Hepatobiliary Cancers. J Gastroenterol (2020) 55(1):1526. doi: 10.1007/s00535-019-01620-7

66. Liu X, Feng Y, Xu J, Shi Y, Yang J, Zhang R, et al. Combination of MAPK Inhibition With Photothermal Therapy Synergistically Augments the AntiTumor Efficacy of Immune Checkpoint Blockade. J Control Release (2021) 332:194-209. doi: 10.1016/j.jconrel.2021.02.020

67. Xu G, Luo Y, Wu W, Liu X, Yu X, Bao Y, et al. The Evolution of Acquired Resistance to BRAFV600E Kinase Inhibitor Is Sustained by IGF1-Driven Tumor Vascular Remodeling. J Invest Dermatol (2021) S0022-202X (21):01669-9. doi: 10.1016/j.jid.2021.07.162

68. Brahmer JR, Drake CG, Wollner I, Powderly JD, Picus J, Sharfman WH, et al. Phase I Study of Single-Agent Anti-Programmed Death-1 (MDX-1106) in Refractory Solid Tumors: Safety, Clinical Activity, Pharmacodynamics, and Immunologic Correlates. J Clin Oncol (2010) 28(19):3167-75. doi: 10.1200/JCO.2009.26.7609

69. Fuchs CS, Doi T, Jang RW, Muro K, Satoh T, Machado M, et al. Safety and Efficacy of Pembrolizumab Monotherapy in Patients With Previously Treated Advanced Gastric and Gastroesophageal Junction Cancer: Phase 2 Clinical KEYNOTE-059 Trial. JAMA Oncol (2018) 4(5):e180013. doi: 10.1001/jamaoncol.2018.0013

70. Francis DM, Manspeaker MP, Schudel A, Sestito LF, O'Melia MJ, Kissick HT, et al. Blockade of Immune Checkpoints in Lymph Nodes Through Locoregional Delivery Augments Cancer Immunotherapy. Sci Transl Med (2020) 12(563):eaay3575. doi: 10.1126/scitranslmed.aay3575

71. El-Khoueiry AB, Sangro B, Yau T, Crocenzi TS, Kudo M, Hsu C, et al. Nivolumab in Patients With Advanced Hepatocellular Carcinoma (CheckMate 040): An Open-Label, non-Comparative, Phase 1/2 Dose Escalation and Expansion Trial. Lancet (2017) 389(10088):2492-502. doi: 10.1016/S0140-6736(17)31046-2

72. Diaz Beveridge R, Alcolea V, Aparicio J, Segura Á, García J, Corbellas M, et al. Management of Advanced Pancreatic Cancer With Gemcitabine Plus Erlotinib: Efficacy and Safety Results in Clinical Practice. JOP (2014) 15 (1):19-24. doi: 10.6092/1590-8577/1570

73. Cardin DB, Goff L, Li CI, Shyr Y, Winkler C, DeVore R, et al. Phase II Trial of Sorafenib and Erlotinib in Advanced Pancreatic Cancer. Cancer Med (2014) 3(3):572-9. doi: 10.1002/cam4.208

74. Llosa NJ, Cruise M, Tam A, Wicks EC, Hechenbleikner EM, Taube JM, et al. The Vigorous Immune Microenvironment of Microsatellite Instable Colon Cancer is Balanced by Multiple Counter-Inhibitory Checkpoints. Cancer Discov (2015) 5(1):43-51. doi: 10.1158/2159-8290.CD-14-0863

75. Biasci D, Smoragiewicz M, Connell CM, Wang Z, Gao Y, Thaventhiran JED, et al. CXCR4 Inhibition in Human Pancreatic and Colorectal Cancers Induces an Integrated Immune Response. Proc Natl Acad Sci U S A (2020) 117(46):28960-70. doi: 10.1073/pnas.2013644117

76. Overman MJ, McDermott R, Leach JL, Lonardi S, Lenz HJ, Morse MA, et al. Nivolumab in Patients With Metastatic DNA Mismatch Repair-Deficient or Microsatellite Instability-High Colorectal Cancer (CheckMate 142): An Open-Label, Multicentre, Phase 2 Study. Lancet Oncol (2017) 18(9):118291. doi: 10.1016/S1470-2045(17)30422-9
77. Han X, Yang J, Luo J, Chen P, Zhang Z, Alu A, et al. Application of CTBased Radiomics in Discriminating Pancreatic Cystadenomas From Pancreatic Neuroendocrine Tumors Using Machine Learning Methods. Front Oncol (2021) 11:606677. doi: 10.3389/fonc.2021.606677

78. Cohen R, Hain E, Buhard O, Guilloux A, Bardier A, Kaci R, et al. Association of Primary Resistance to Immune Checkpoint Inhibitors in Metastatic Colorectal Cancer With Misdiagnosis of Microsatellite Instability or Mismatch Repair Deficiency Status. JAMA Oncol (2019) 5(4):551-5. doi: 10.1001/jamaoncol.2018.4942

79. Gurjao C, Liu D, Hofree M, AlDubayan SH, Wakiro I, Su MJ, et al. Intrinsic Resistance to Immune Checkpoint Blockade in a Mismatch Repair-Deficient Colorectal Cancer. Cancer Immunol Res (2019) 7(8):1230-6. doi: 10.1158/ 2326-6066.CIR-18-0683

80. Venderbosch S, Nagtegaal ID, Maughan TS, Smith CG, Cheadle JP, Fisher D, et al. Mismatch Repair Status and BRAF Mutation Status in Metastatic Colorectal Cancer Patients: A Pooled Analysis of the CAIRO, CAIRO2, COIN, and FOCUS Studies. Clin Cancer Res (2014) 20(20):5322-30. doi: 10.1158/1078-0432.CCR-14-0332

81. Yu C, Luo D, Yu J, Zhang M, Zheng X, Xu G, et al. Genome-Wide CRISPRCas9 Knockout Screening Identifies GRB7 as a Driver for MEK Inhibitor Resistance in KRAS Mutant Colon Cancer. Oncogene (2021). doi: 10.1038/ s41388-021-02077-w

82. Cheng Y, Han X, Mo F, Zeng H, Zhao Y, Wang H, et al. Apigenin Inhibits the Growth of Colorectal Cancer Through Down-Regulation of E2F1/3 by miRNA-215-5p. Phytomedicine (2021) 89:153603. doi: 10.1016/ j.phymed.2021.153603

83. Lee JJ, Chu E. Recent Advances in the Clinical Development of Immune Checkpoint Blockade Therapy for Mismatch Repair Proficient (pMMR)/ non-MSI-H Metastatic Colorectal Cancer. Clin Colorectal Cancer (2018) 17 (4):258-73. doi: 10.1016/j.clcc.2018.06.004

84. Sun L, Huang S, Li D, Mao Y, Wang Y, Wu J. Efficacy and Safety of Fruquintinib Plus PD-1 Inhibitors Versus Regorafenib Plus PD-1 Inhibitors in Refractory Microsatellite Stable Metastatic Colorectal Cancer. Front Oncol (2021) 11:754881. doi: 10.3389/fonc.2021.754881

85. Zhang Y, Li J, Wang B, Chen T, Chen Y, Ma W. LDH-A Negatively Regulates dMMR in Colorectal Cancer. Cancer Sci (2021) 112(8):3050-63. doi: $10.1111 /$ cas. 15020

86. Loi S, Dushyanthen S, Beavis PA, Salgado R, Denkert C, Savas P, et al. RAS/ MAPK Activation Is Associated With Reduced Tumor-Infiltrating Lymphocytes in Triple-Negative Breast Cancer: Therapeutic Cooperation Between MEK and PD-1/PD-L1 Immune Checkpoint Inhibitors. Clin Cancer Res (2016) 22(6):1499-509. doi: 10.1158/1078-0432.CCR-15-1125

87. Yu C, Zhang M, Song J, Zheng X, Xu G, Bao Y, et al. Integrin-Src-YAP1 Signaling Mediates the Melanoma Acquired Resistance to MAPK and PI3K/ mTOR Dual Targeted Therapy. Mol Biomed (2020) 1(1):12. doi: 10.1186/ s43556-020-00013-0

88. Liu X, Feng Y, Xu G, Chen Y, Luo Y, Song J, et al. MAPK-Targeted Drug Delivered by a pH-Sensitive MSNP Nanocarrier Synergizes With PD-1 Blockade in Melanoma Without T-Cell Suppression. Adv Funct Mater (2019) 1806916. doi: 10.1002/adfm.201806916

89. Chalabi M, Fanchi LF, Dijkstra KK, Van den Berg JG, Aalbers AG, Sikorska $\mathrm{K}$, et al. Neoadjuvant Immunotherapy Leads to Pathological Responses in MMR-Proficient and MMR-Deficient Early-Stage Colon Cancers. Nat Med (2020) 26(4):566-76. doi: 10.1038/s41591-020-0805-8

90. Tauriello DVF, Palomo-Ponce S, Stork D, Berenguer-Llergo A, BadiaRamentol J, Iglesias M, et al. Tgf $\beta$ Drives Immune Evasion in Genetically Reconstituted Colon Cancer Metastasis. Nature (2018) 554(7693):538-43. doi: 10.1038/nature25492

91. Yoo CB, Jones PA. Epigenetic Therapy of Cancer: Past, Present and Future. Nat Rev Drug Discovery (2006) 5(1):37-50. doi: 10.1038/nrd1930

92. Pan Y, Liu G, Zhou F, Su B, Li Y. DNA Methylation Profiles in Cancer Diagnosis and Therapeutics. Clin Exp Med (2018) 18(1):1-14. doi: 10.1007/ s10238-017-0467-0

93. Xie VK, Li Z, Yan Y, Jia Z, Zuo X, Ju Z, et al. DNA-Methyltransferase 1 Induces Dedifferentiation of Pancreatic Cancer Cells Through Silencing of Krüppel-Like Factor 4 Expression. Clin Cancer Res (2017) 23(18):5585-97. doi: 10.1158/1078-0432.CCR-17-0387 
94. Jian D, Wang Y, Jian L, Tang H, Rao L, Chen K, et al. METTL14 Aggravates Endothelial Inflammation and Atherosclerosis by Increasing FOXO1 N6Methyladeosine Modifications. Theranostics (2020) 10(20):8939-56. doi: $10.7150 /$ thno. 45178

95. Tu Z, Wu L, Wang P, Hu Q, Tao C, Li K, et al. N6-MethylandenosineRelated lncRNAs Are Potential Biomarkers for Predicting the Overall Survival of Lower-Grade Glioma Patients. Front Cell Dev Biol (2020) 8:642. doi: $10.3389 /$ fcell.2020.00642

96. Cao G, Li HB, Yin Z, Flavell RA. Recent Advances in Dynamic M6a RNA Modification. Open Biol (2016) 6(4):160003. doi: 10.1098/rsob.160003

97. Schöller E, Weichmann F, Treiber T, Ringle S, Treiber N, Flatley A, et al. Interactions, Localization, and Phosphorylation of the M6a Generating METTL3-METTL14-WTAP Complex. RNA (2018) 24(4):499-512. doi: 10.1261/rna.064063.117

98. Li A, Chen YS, Ping XL, Yang X, Xiao W, Yang Y, et al. Cytoplasmic M6a Reader YTHDF3 Promotes mRNA Translation. Cell Res (2017) 27(3):444-7. doi: $10.1038 / \mathrm{cr} .2017 .10$

99. Alarcón CR, Goodarzi H, Lee H, Liu X, Tavazoie S, Tavazoie SF. HNRNPA2B1 Is a Mediator of M(6)A-Dependent Nuclear RNA Processing Events. Cell (2015) 162(6):1299-308. doi: 10.1016/j.cell.2015.08.011

100. Wu R, Yao Y, Jiang Q, Cai M, Liu Q, Wang Y, et al. Epigallocatechin Gallate Targets FTO and Inhibits Adipogenesis in an mRNA M6a-YTHDF2Dependent Manner. Int J Obes (Lond) (2018) 42(7):1378-88. doi: 10.1038/ s41366-018-0082-5

101. Relier S, Ripoll J, Guillorit H, Amalric A, Achour C, Boissière F, et al. FTOMediated Cytoplasmic m6Am Demethylation Adjusts Stem-Like Properties in Colorectal Cancer Cell. Nat Commun (2021) 12(1):1716. doi: 10.1038/ s41467-021-21758-4

102. Tang C, Klukovich R, Peng H, Wang Z, Yu T, Zhang Y, et al. ALKBH5Dependent M6a Demethylation Controls Splicing and Stability of Long 3'UTR mRNAs in Male Germ Cells. Proc Natl Acad Sci U S A (2018) 115(2): E325-33. doi: 10.1073/pnas.1717794115

103. Riley RS, June CH, Langer R, Mitchell MJ. Delivery Technologies for Cancer Immunotherapy. Nat Rev Drug Discov (2019) 18(3):175-96. doi: 10.1038/ s41573-018-0006-z

104. Bivona TG, Doebele RC. A Framework for Understanding and Targeting Residual Disease in Oncogene-Driven Solid Cancers. Nat Med (2016) 22 (5):472-8. doi: 10.1038/nm.4091

105. Chen Z, Shi T, Zhang L, Zhu P, Deng M, Huang C, et al. Mammalian Drug Efflux Transporters of the ATP Binding Cassette (ABC) Family in Multidrug Resistance: A Review of the Past Decade. Cancer Lett (2016) 370(1):153-64. doi: 10.1016/j.canlet.2015.10.010

106. Joyce H, McCann A, Clynes M, Larkin A. Influence of Multidrug Resistance and Drug Transport Proteins on Chemotherapy Drug Metabolism. Expert Opin Drug Metab Toxicol (2015) 11(5):795-809. doi: 10.1517/17425255. 2015.1028356

107. Wang Y, Fang Z, Hong M, Yang D, Xie W. Long-Noncoding RNAs (lncRNAs) in Drug Metabolism and Disposition, Implications in Cancer Chemo-Resistance. Acta Pharm Sin B (2020) 10(1):105-12. doi: 10.1016/j.apsb.2019.09.011

108. Zhitomirsky B, Assaraf YG. Lysosomes as Mediators of Drug Resistance in Cancer. Drug Resist Updat (2016) 24:23-33. doi: 10.1016/j.drup.2015.11.004

109. Li B, Jiang J, Assaraf YG, Xiao H, Chen ZS, Huang C. Surmounting Cancer Drug Resistance: New Insights From the Perspective of N6-Methyladenosine RNA Modification. Drug Resist Updat (2020) 53:100720. doi: 10.1016/ j.drup.2020.100720

110. Chen H, Yao J, Bao R, Dong Y, Zhang T, Du Y, et al. Cross-Talk of Four Types of RNA Modification Writers Defines Tumor Microenvironment and Pharmacogenomic Landscape in Colorectal Cancer. Mol Cancer (2021) 20 (1):29. doi: 10.1186/s12943-021-01322-w

111. Yang F, Jin H, Que B, Chao Y, Zhang H, Ying X, et al. Dynamic M6a mRNA Methylation Reveals the Role of METTL3-M6a-CDCP1 Signaling Axis in Chemical Carcinogenesis. Oncogene (2019) 38(24):4755-72. doi: 10.1038/ s41388-019-0755-0

112. Wang H, Hu X, Huang M, Liu J, Gu Y, Ma L, et al. Mettl3-Mediated mRNA M6a Methylation Promotes Dendritic Cell Activation. Nat Commun (2019) 10(1):1898. doi: 10.1038/s41467-019-09903-6

113. Dong L, Chen C, Zhang Y, Guo P, Wang Z, Li J, et al. The Loss of RNA N6Adenosine Methyltransferase Mettl14 in Tumor-Associated Macrophages
Promotes CD8+ T Cell Dysfunction and Tumor Growth. Cancer Cell (2021) 39(7):945-57.e10. doi: 10.1016/j.ccell.2021.04.016

114. Wang S, Gan M, Chen C, Zhang Y, Kong J, Zhang H, et al. Methyl CpG Binding Protein 2 Promotes Colorectal Cancer Metastasis by Regulating N6 -Methyladenosine Methylation Through Methyltransferase-Like 14. Cancer Sci (2021) 112(8):3243-54. doi: 10.1111/cas.15011

115. Zhang B, Wu Q, Li B, Wang D, Wang L, Zhou YL. M6a Regulator-Mediated Methylation Modification Patterns and Tumor Microenvironment Infiltration Characterization in Gastric Cancer. Mol Cancer (2020) 19 (1):53. doi: 10.1186/s12943-020-01170-0

116. Zheng Y, Wang Y, Liu Y, Xie L, Ge J, Yu G, et al. N6-Methyladenosine Modification of PTTG3P Contributes to Colorectal Cancer Proliferation via YAP1. Front Oncol (2021) 11:669731. doi: 10.3389/fonc.2021.669731

117. Liu X, Su K, Sun X, Jiang Y, Wang L, Hu C, et al. Sec62 Promotes Stemness and Chemoresistance of Human Colorectal Cancer Through Activating Wnt/B-Catenin Pathway. J Exp Clin Cancer Res (2021) 40(1):132. doi: 10.1186/s13046-021-01934-6

118. Chen C, Yuan W, Zhou Q, Shao B, Guo Y, Wang W, et al. N6Methyladenosine-Induced Circ1662 Promotes Metastasis of Colorectal Cancer by Accelerating YAP1 Nuclear Localization. Theranostics (2021) 11(9):4298-315. doi: 10.7150/thno.51342

119. He RZ, Jiang J, Hu X, Lei M, Li J, Luo W, et al. Stabilization of UCA1 by N6Methyladenosine RNA Methylation Modification Promotes Colorectal Cancer Progression. Cancer Cell Int (2021) 21(1):616. doi: 10.1186/s12935021-02288-x

120. Chen X, Xu M, Xu X, Zeng K, Liu X, Sun L, et al. METTL14 Suppresses CRC Progression via Regulating N6-Methyladenosine-Dependent Primary miR-375 Processing. Mol Ther (2020) 28(2):599-612. doi: 10.1016/j.ymthe.2019.11.016

121. Wang L, Hui H, Agrawal K, Kang Y, Li N, Tang R, et al. M6 A RNA Methyltransferases METTL3/14 Regulate Immune Responses to Anti-PD-1 Therapy. EMBO J (2020) 39(20):e104514. doi: 10.15252/embj.2020104514

122. Zhang Z, Gao Q, Wang S. Kinase Gsk3 $\beta$ Functions as a Suppressor in Colorectal Carcinoma Through the FTO-Mediated MZF1/c-Myc Axis. J Cell Mol Med (2021) 25(5):2655-65. doi: 10.1111/jcmm.16291

123. Ruan DY, Li T, Wang YN, Meng Q, Li Y, Yu K, et al. FTO Downregulation Mediated by Hypoxia Facilitates Colorectal Cancer Metastasis. Oncogene (2021) 40(33):5168-81. doi: 10.1038/s41388-021-01916-0

124. Yang S, Wei J, Cui YH, Park G, Shah P, Deng Y, et al. M6a mRNA Demethylase FTO Regulates Melanoma Tumorigenicity and Response to Anti-PD-1 Blockade. Nat Commun (2019) 10(1):2782. doi: 10.1038/s41467019-10669-0

125. Li N, Kang Y, Wang L, Huff S, Tang R, Hui H, et al. ALKBH5 Regulates AntiPD-1 Therapy Response by Modulating Lactate and Suppressive Immune Cell Accumulation in Tumor Microenvironment. Proc Natl Acad Sci U S A (2020) 117(33):20159-70. doi: 10.1073/pnas.1918986117

126. Kuai D, Zhu S, Shi H, Yang R, Liu T, Liu H, et al. Aberrant Expression of M6a mRNA Methylation Regulators in Colorectal Adenoma and Adenocarcinoma. Life Sci (2021) 273:119258. doi: 10.1016/j.lfs.2021.119258

127. Chen P, Liu XQ, Lin X, Gao LY, Zhang S, Huang X. Targeting YTHDF1 Effectively Re-Sensitizes Cisplatin-Resistant Colon Cancer Cells by Modulating GLS-Mediated Glutamine Metabolism. Mol Ther Oncolytics (2021) 20:228-39. doi: 10.1016/j.omto.2021.01.001

128. Li H, Zhang N, Jiao X, Wang C, Sun W, He Y, et al. Downregulation of microRNA-6125 Promotes Colorectal Cancer Growth Through YTHDF2Dependent Recognition of N6-Methyladenosine-Modified GSK3 $\beta$. Clin Transl Med (2021) 11(10):e602. doi: 10.1002/ctm2.602

129. Tsuchiya K, Yoshimura K, Inoue Y, Iwashita Y, Yamada H, Kawase A, et al. YTHDF1 and YTHDF2 are Associated With Better Patient Survival and an Inflamed Tumor-Immune Microenvironment in non-Small-Cell Lung Cancer. Oncoimmunology (2021) 10(1):1962656. doi: 10.1080/2162402X.2021.1962656

Conflict of Interest: The authors declare that the research was conducted in the absence of any commercial or financial relationships that could be construed as a potential conflict of interest.

Publisher's Note: All claims expressed in this article are solely those of the authors and do not necessarily represent those of their affiliated organizations, or those of the publisher, the editors and the reviewers. Any product that may be evaluated in 
this article, or claim that may be made by its manufacturer, is not guaranteed or endorsed by the publisher.

Copyright (๑ 2022 Tong, Wei, Smith and Huang. This is an open-access article distributed under the terms of the Creative Commons Attribution License
(CC BY). The use, distribution or reproduction in other forums is permitted, provided the original author(s) and the copyright owner(s) are credited and that the original publication in this journal is cited, in accordance with accepted academic practice. No use, distribution or reproduction is permitted which does not comply with these terms. 